\title{
Darstellung von Harnstoff durch Oxydation von Eiweiss mit Permanganat nach A. Jolles. \\ Von \\ Emil Abderhalden.
}

(Aus dem I. Chemischen Institut der Universität Berlin.)

(Der Redaction zugegangen am 5. März 1903.)

Die Angabe von A. Jolles, ${ }^{1}$ ) dass bei der Oxydation des Eiweisses mit Permanganat in schwefelsaurer Lösung der Stickstoff fast quantitativ als Harnstoff erhalten wird, ist bereits von F. N. Schulz ${ }^{2}$ ) einer Nachprüfung unterzogen worden. Schulz konnte die Angaben von Jolles nicht bestätigen. Jolles ${ }^{3}$ ) glaubt den negativen Ausfall der Nachprüfung von Schulz durch ein seiner Methode nicht genau entsprechendes Verfahren erklären zu können. Es schien mir bei der weittragenden Bedeutung, den eine Bestätigung der Befunde von Jolles für die ganze Auffassung des Eiweissmoleküls haben würde, erwünscht, die Angaben Jolles' unter peinlicher Innehaltung seiner Vorschriften zu kontrolliren.

Ausgeführt wurden die Versuche an Casein. 0,5 g Casein (Hammarsten)wurden in $400 \mathrm{ccm}$ destillierten Wassers suspendirt, $10 \mathrm{ccm}$ concentrirte Schwefelsäure vom specifischen Gewichte 1,84 zugesetzt, auf dem Drahtnetze erwärmt, und nun aus einer Bürette eine $0,4 \%$ ige Permanganatlösung vorsichtig zugesetzt. Die Oxydation dauerte zweimal ca. 10 Stunden. Das Volumen

1) A. Jolles, Beiträge zur Kenntniss der Eiweisskörper, Diese Zeitschrift, Bd. XXXII, 1901, S. 364.

2) F. N. Schulz, Ueber Darstellung von Harnstoff durch Oxydation von Eiweiss nach Jolles, Diese Zeitschrift, Bd. XXXIII, 1901, S. 363.

3) A. Jolles, Ueber Darstellung von Harnstoff durch Oxydation von Eiweiss. Diese Zeitschrift, Bd. XXXIV, 1901/02, S. 28. 
der Flüssigkeit wurde stets beibehalten. Verbraucht wurden ca. 400-425 ccm der Permanganatlösung. Der Nachweis des Harnstoffs wurde in vier Versuchen unter peinlich genauer Befolgung der Jolles'schen Vorschriften versucht. In keinem einzigen Falle konnte Harnstoff mit Sicherheit nachgewiesen werden.

Die von Jolles angegebene Methode ist sehr umständlich und zeitraubend. Ihre Ausführung erschwert eine "quantitative» Bestimmung durch die grosse Zahl der auszuführenden Operationen sehr. Es wurde deshalb auf eine einfachere Weise die etwa erfolgte Harnstoffbildung nachzuweisen versucht. Die Oxydation wurde der Vorschrift von Jolles folgend zu Ende geführt und hierauf die Lösung mit Natronlauge unter Kühlung so lange versetzt, bis Mangan auszufallen begann. Hierauf wurde die Lösung bei $10 \mathrm{~mm}$ Druck eingeengt, die eingeengte Flüssigkeit mit absolutem Alkohol versetzt, die ausgeschiedenen Salze abfiltrirt, und das Filtrat weiter eingeengt. Nach nochmaliger Fällung mit absolutem Alkohol wurde die Mutterlauge im Vacuum zur Trockene eingedampft. Der Rückstand wurde hierauf mit absolutem Alkohol ausgekocht. Nach dem Verdunsten des Alkohols verblieb eine Spur einer syrupösen Substanz. Sämmtliche Reactionen auf Harnstoff fielen negativ aus. ${ }^{1}$ )

Aus den vorliegenden Versuchen ergibt sich, dass bei peinlicher Innehaltung der von Jolles mitgeteilten Methode Harnstoff mit Sicherheit nicht erhalten werden kann. Ich bitte deshalb Herrn A. Jolles, die Methode, mit welcher es ihm gelang, den Stickstoff verschiedener Eiweisskörper fast quantitativ als Harnstoff zu gewinnen, so wiederzugeben, dass es auch Anderen gelingt, seine Resultate zu erhalten.

1) Dass mit der angewandten Methode etwa vorhandener Harnstoff quantitativ nachweisbar wäre, lehrte der folgende Kontrollversuch. Es wurden zu einer nach der Jolles'schen Vorschrift oxydirten Eiweisslösung $(0,5 \mathrm{~g}$ Casein) $0,05 \mathrm{~g}$ Harnstoff zugesetzt. Wieder erhalten wurden $0,046 \mathrm{~g}$. 